\title{
Bio-compatible polymer coatings using low temperature, atmospheric pressure plasma
}

\author{
S. Farhat, M. Gilliam, A. Zand \& M. Rabago-Smith \\ Department of Chemistry, Biochemistry, and Chemical Engineering, \\ Kettering University, Michigan, USA
}

\begin{abstract}
Current research has been dedicated to investigating the viability of atmospheric pressure plasmas for use in coating technology. In addition to being more cost effective and efficient, atmospheric pressure plasma offers a more streamlined process, as it can be employed directly into the production line. Atmospheric pressure plasma has been used in applications including biocompatibility, hydrophilicity/hydrophobicity, and coating with antibacterial films.

Polyethylene is used as a biocompatible surface for ball and socket joint replacements, which are under constant wear. Atmospheric pressure plasma treatment was used to change the surface chemistry by grafting various biocompatible polymers to the polyethylene surface, as methods of providing wear resistance as well as providing a self-lubricating surface. The organic coatings included biocompatible polymers, such as poly(2hydroxyethylmethacrylate), polyethylenimine, and polyethylene glycol. Low temperature, atmospheric pressure plasma was used, along with an in-house constructed spray delivery system, to coat high density polyethylene substrates. Coatings were characterized with Fourier transform infrared spectroscopy (FTIR), contact angle analysis, and adhesion testing. A significant decrease in contact angle was noted for various coatings produced with this method, indicating an increased wettability. Plasma processing conditions, specifically the pretreatment of the substrate and the input power, greatly affected the adhesion and uniformity of the polymerized layer.
\end{abstract}

Keywords: atmospheric pressure plasma, coatings, hydrophilicity. 


\section{Introduction}

\subsection{Biocompatible coatings and applications}

Medical devices and biomedical implants may be fabricated from metal, ceramic, plastics, or a combination. Medical devices used inside the body, such as artificial implants, can release particles into the body due to wear or erosion. The release of such particles may result in an immune response, infection, inflammation, destruction of bone and muscle, or other negative effects on the body [1-3]. For example, metal hip replacements have a high rate of failure and the rubbing between the ball and cup can cause material to break off and seep into tissue, thereby, causing complications. Since the interface between the implant and the body is essential for performance, choice of materials for this interface is critical for successful application in such devices. Polymer coatings can be designed to have specific chemical, mechanical, and physical properties for uses in different medical device applications. To be successful for these applications, coatings must be not only bio-compatible, but must also be well adhered to the implant or device itself and be strong enough to withstand movement expected during usage as well as the environment. These materials can be applied to implants or other devices using solvents or aqueous solutions or in vapour phase, including using plasma deposition [1-4].

\subsection{Plasma applications}

Plasmas play a critical role in many areas of applications including surface cleaning, coatings, printing, painting, adhesive bonding, as well as automotive and aerospace industries. When plasma contacts material surfaces, the additional energy can be transferred and reactions can take place on the surface, resulting in altered surface properties depending on the plasma conditions. Atmospheric pressure plasmas offer an interesting alternative to typical vacuum plasmas (and even wet chemistry treatments) due to the ease of in-line processing, lower costs, quicker processing, and decreased environmental effects. The atmospheric pressure plasma may be generated by any feasible method of generating plasma under atmospheric conditions, such as electrical ignition using direct current (DC), alternating current (AC), or pulsed current, among others. The work presented here was completed using atmospheric pressure plasma delivered by a jet through a nozzle to the substrate to produce a coating $[4,5]$.

\subsection{Atmospheric pressure plasma for coatings}

For the coating process, atmospheric pressure plasma is used to activate the surface of the substrate to create attractive or radical sites for bonding to the chemical precursor for the coating itself. Simultaneously, the plasma reacts with the chemical precursors to partially dissociate the molecules and graft them to the surface of the substrate as well as cross-link the precursor molecules to form a coating. The gas for the plasma may be any gas that can be ionized by a plasma generator that does not form a solid coating, such as nitrogen, oxygen, 
noble gases, carbon dioxide, or any combination. The plasma conditions, which are controllable and variable during the coating process, include the type of gas, power, the flow rate of gas, the distance from the substrate, and the rate of scanning. The optimum plasma conditions may differ for each combination of substrate, chemical precursor, and final application of the article. In general, the set of plasma conditions are tailored to meet the desired wear resistance, adhesion, durability, biocompatibility, hydrophilicity, and other desired properties of the applied coating.

\section{Experimental methods}

\subsection{Atmospheric pressure plasma process}

The atmospheric pressure plasma jet system was a PlasmaTreat AS400 system utilizing Openair $^{\mathbb{R}}$ technology. The plasma is generated using compressed air as the ionization gas, with frequencies ranging from $19-21 \mathrm{kHz}$, and input power of 750-1400W.

Figure 1 shows the general set-up for the coating and treating process of the substrates. The biopolymer precursors used for this study were delivered in liquid form to the substrate surface prior to plasma exposure in one step (as shown in Figure 1). The plasma jet was designed to scan the material in the $\mathrm{x}$ and $z$ direction; the distance between the plasma nozzle and the substrate (L) was set before experimental runs. The speed at which the plasma jet and spray delivery system for the precursor scanned the substrate (in the x-direction) was controlled by a computer interface.

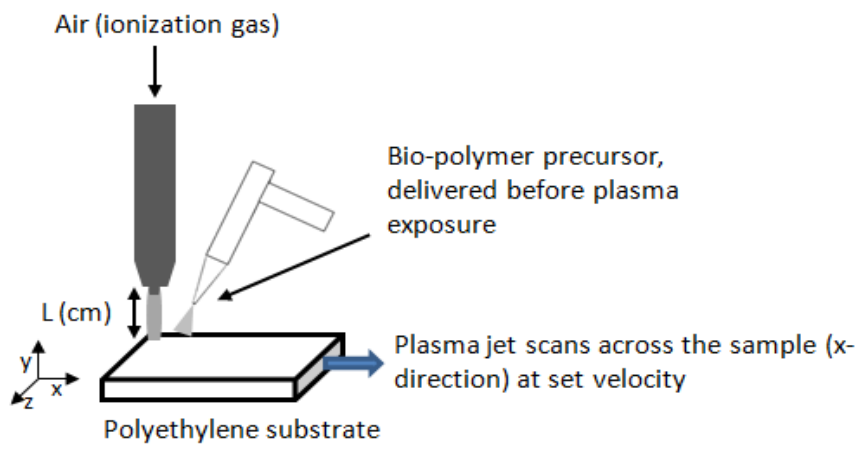

Figure 1: Coating process using atmospheric pressure plasma.

The variables analysed for this study included the effect of pre-treating the substrates, scanning speed of the plasma jet, and input power for the plasma. For the pre-treatment process, the substrates (polyethylene square plaques, dimensions $5 \mathrm{~cm} \times 5 \mathrm{~cm}$ ) were treated using air plasma at a distance of $2.5 \mathrm{~cm}$ from the plasma nozzle. The pre-treatment conditions for the plasma are shown in Table 1. Pre-treatment could enhance adhesion of the grafted polymer by creating active bonding sites on the surface of the polyethylene. 
Table 1: Plasma settings for pre-treatment.

\begin{tabular}{lc}
\hline Parameter & Setting \\
\hline \hline Power $(\mathrm{W})$ & 1250 \\
Frequency $(\mathrm{kHz})$ & 21 \\
Air flow rate $(\mathrm{L} / \mathrm{min})$ & 30 \\
Substrate scan speed (m/min) & 15 \\
Substrate distance, L (cm) & 2.5 \\
\hline
\end{tabular}

\subsection{Chemical precursors}

The chemical precursors used for this study were biocompatible polymers and included poly(2-hydroxyethylmethacrylate) and its monomer (hydroxyethylmethacrylate), polyethylenimine, and polyethylene glycol (Sigma Aldrich). Each polymer was dissolved in water; concentrations for each can be found in Table 2.

Table 2: Chemical precursors and concentrations for solutions.

\begin{tabular}{lcc}
\hline Precursor & Abbreviation & Concentration \\
\hline \hline Poly(2-hydroxyethylmethacrylate) & PHEMA & $1.5 \mathrm{wt} \%$ in $\mathrm{H}_{2} \mathrm{O}$ \\
Polyethylenimine & PEI & $8-16 \mathrm{wt} \%$ in $\mathrm{H}_{2} \mathrm{O}$ \\
Polyethylene glycol & PEG & $25 \mathrm{wt} \%$ in $\mathrm{H}_{2} \mathrm{O}$ \\
Hydroxyethylmethacrylate & HEMA & $50 \mathrm{wt} \%$ in PHEMA solution \\
\hline
\end{tabular}

The precursors were delivered via a pressurized spray system at an angle of 45 degrees from the plasma jet at an average flow rate of $0.1 \mathrm{~g} / \mathrm{min}$. The flow rate of precursor was controlled by the pressure to the spray system. The spray system ensured an even coating on the substrate before exposure to plasma; each substrate was coated 3 times. The distance from the plasma nozzle to the substrate for these coatings was kept constant at $2.5 \mathrm{~cm}$. A substrate distance less than $2 \mathrm{~cm}$ could result in deformation of the substrate due to the heat from the plasma.

\subsection{Characterization}

Fourier transform infrared (FTIR) spectroscopy was used to interpret the surface bonds of the plasma grafted coatings. A Nicolet 6780 FT-IR by Thermo Scientific was used for this study. Drop contact angle was analyzed using computer software; both water contact angle and albumin contact angle were measured using computer software to analyze droplets. A cross-cut tape test following ASTM D3359 was used to measure adhesion of the coatings to the PE substrates. 


\section{Results and discussion}

\subsection{FTIR}

FTIR results for samples coated with all four liquid precursors using the technique shown previously (Figure 1) are displayed in Figure 2.

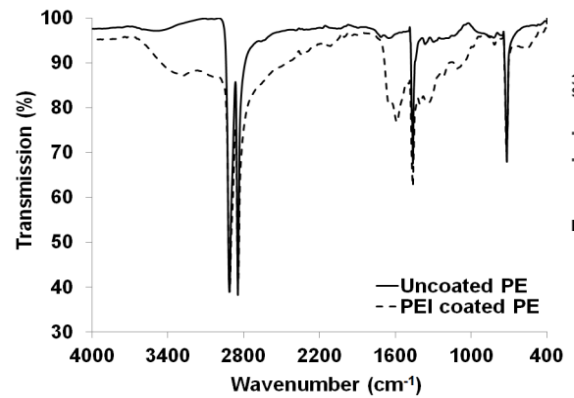

(a)

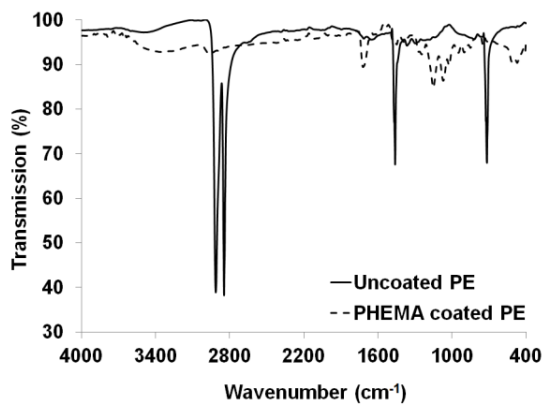

(c)

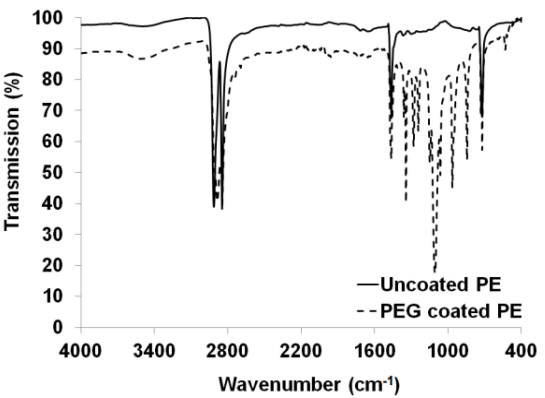

(b)

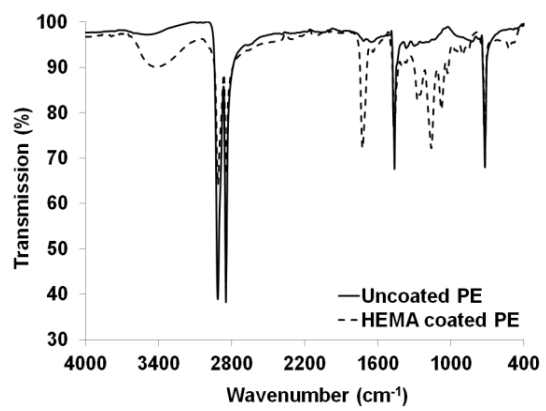

(d)

Figure 2: $\quad$ FTIR spectra for (a) PEI coatings, (b) PEG coatings, (c) PHEMA coatings, and (d) HEMA coatings on polyethylene compared to uncoated PE

For each of these coatings, the settings were $1400 \mathrm{~W}$ for input power, pretreated PE substrates, and $15 \mathrm{~m} / \mathrm{min}$ scan speed. As shown in each plot, new surface chemistry was noted when comparing the uncoated polyethylene to the plasma coated polyethylene. For each liquid precursor, an - $\mathrm{OH}$ bond was noted $\left(3400 \mathrm{~cm}^{-1}\right)$; this hydrophilicity was verified with contact angle as well. Figure 2(a) featured a much more broad peak in the $3400-3500 \mathrm{~cm}^{-1}$ which was indicative of the $-\mathrm{NH}$ and $-\mathrm{OH}$ bonds present in the PEI coating. The PHEMA and HEMA coatings as shown in Figures 2(c) and (d) showed a carbonyl peak $(\mathrm{C}=\mathrm{O})$ around $1600 \mathrm{~cm}^{-1}$, indicative of the precursor chemistry seen in PHEMA. The more intense carbonyl peak for the HEMA addition to the coatings could be due to a more uniformly cross-linked layer. 
Concentration of the precursor solution did not affect the surface chemistry considerably when comparing the FTIR of the surfaces; however, since FTIR does not provide quantitative analysis, further testing would be necessary to understand these effects.

\subsection{Adhesion}

A cross-cut tape test following ASTM D3359 was used to measure adhesion of the coatings to the PE substrates. A crosshatch is marked in the coating and tape is applied evenly to the crosshatch for 90 seconds before being removed. Coatings that passed adhesion showed no peeling in any squares of the crosshatch after the tape was pulled; while coatings that failed adhesion showed peeling in one or more of the squares of the crosshatch after the tape was pulled.

For pre-treated substrates all samples coated with PEG passed the adhesion test. For PEI coatings, the coating itself was sometimes tacky due to the high viscosity polymer solution. Coatings of PEI passed when higher input power was used (as shown in Table 3). The substrates for these coatings were pretreated PE.

Table 3: PEI coatings adhesion results.

\begin{tabular}{cccc}
\hline $\begin{array}{c}\text { PEI } \\
\text { concentration }\end{array}$ & $\begin{array}{c}\text { Input } \\
\text { power } \\
(\mathbf{W})\end{array}$ & $\begin{array}{c}\text { Scan } \\
\text { speed } \\
(\mathbf{m} / \mathbf{m i n})\end{array}$ & Adhesion \\
\hline \hline & 1375 & 10 & pass \\
\hline \multirow{2}{*}{ wt.\% in $\mathrm{H}_{2} \mathrm{O}$} & 950 & 10 & fail \\
& 945 & 10 & fail \\
& 1150 & 10 & pass \\
& 1400 & 10 & pass \\
& 900 & 10 & fail \\
& 950 & 10 & fail \\
& 1200 & 10 & pass \\
\hline
\end{tabular}

PHEMA was coated on both pre-treated and non pre-treated substrates - the effect of this was discussed using FTIR analysis as well. Results of adhesion analysis on PHEMA coated samples are shown in Table 4. Adhesion was affected by the pre-treatment as well as the power and scan speed.

Higher scan speed and non pre-treated substrates failed for the lower input power plasma settings. The lower plasma power could decrease the interaction between the precursor and plasma and result in lower quality coatings; the same effect could be attributed to faster scan speeds for the plasma jet. The adhesion results shown above clearly indicate the need for pre-treating substrates before coating. The pre-treatment process provides active bonding sites for the polymer to bond.

Although the lower scan speed could be providing a more uniform and thicker coating, but the bonding of the precursor to the substrate surface will depend on 
Table 4: PHEMA coating adhesion results.

\begin{tabular}{cccc}
\hline $\begin{array}{c}\text { Pre- } \\
\text { treat }\end{array}$ & $\begin{array}{c}\text { Input } \\
\text { power } \\
(\mathbf{W})\end{array}$ & $\begin{array}{c}\text { Scan } \\
\text { speed } \\
(\mathbf{m} / \mathbf{m i n})\end{array}$ & $\begin{array}{c}\text { Initial } \\
\text { adhesion }\end{array}$ \\
\hline \hline yes & 945 & 20 & Pass \\
no & 965 & 20 & Fail \\
yes & 1330 & 10 & Pass \\
no & 1370 & 10 & Pass \\
yes & 1250 & 15 & Pass \\
no & 1330 & 15 & Pass \\
yes & 1250 & 20 & Pass \\
no & 1350 & 20 & Fail \\
yes & 960 & 10 & Pass \\
no & 965 & 10 & Fail \\
yes & 980 & 15 & Pass \\
no & 985 & 15 & Pass \\
\hline
\end{tabular}

other variables like plasma power. This dependence could also have a dependence on the type of precursor and the bond dissociations involved in creating the grafted coating. With this in mind, based on the desired application and polymer used for coating, the plasma parameters would need to be determined through optimization.

\subsection{Contact angle}

Contact angle analysis was performed for the coated samples to assess the compatibility of the surfaces with aqueous solution as in the body. Contact angle measurements were obtained using DI water and in some cases an albumin solution. The results in Figure 3 show that the coatings were significantly hydrophilic, indicating compatibility with aqueous solutions, such as in the body.

The coatings used for contact angle measurements shown in Figure 3 were using plasma input power of $1350 \mathrm{~W}$ and $10 \mathrm{~m} / \mathrm{min}$ scan speed, with three coats of the precursors. PEI showed the most drastic decrease in contact angle, but all coatings created a more wettable surface. Uncoated polyethylene contact angle reduced from 90 degrees to as low as 35 degrees for these coatings. More optimization would be required to tailor each precursor coating for the desired application. When comparing the PHEMA to HEMA coatings, HEMA reduced contact angle even more than PHEMA due to possibly stronger crosslinking with the addition of the monomer to the precursor. 


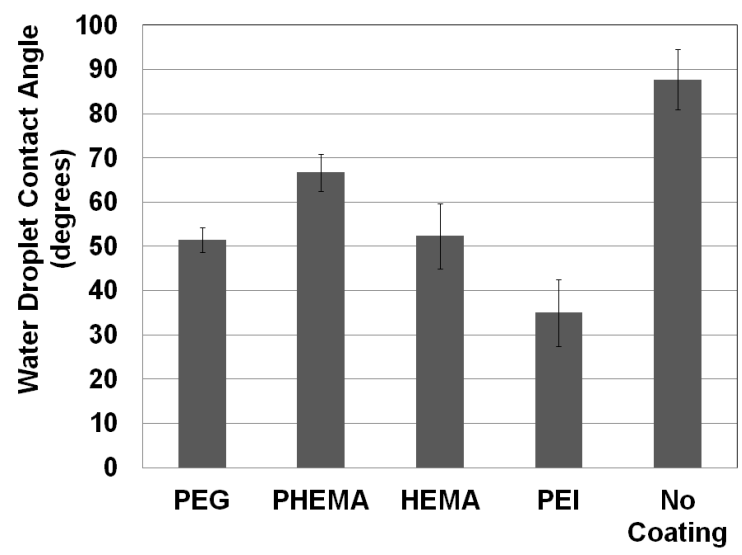

Figure 3: Water droplet contact angle for various coatings compared to uncoated polyethylene. Standard deviation for measurements is shown with error bars.

Figure 4 shows the comparison of contact angle for varying concentration of PEI as the liquid precursor. Little effect on contact angle was noted when comparing varying input power from the plasma. For high power, a slight change in contact angle was noted, but the high standard deviation could indicate that the statistical difference is not significant. Further work is on-going to investigate the relationship between plasma parameters and the quality of the coating and contact angle.

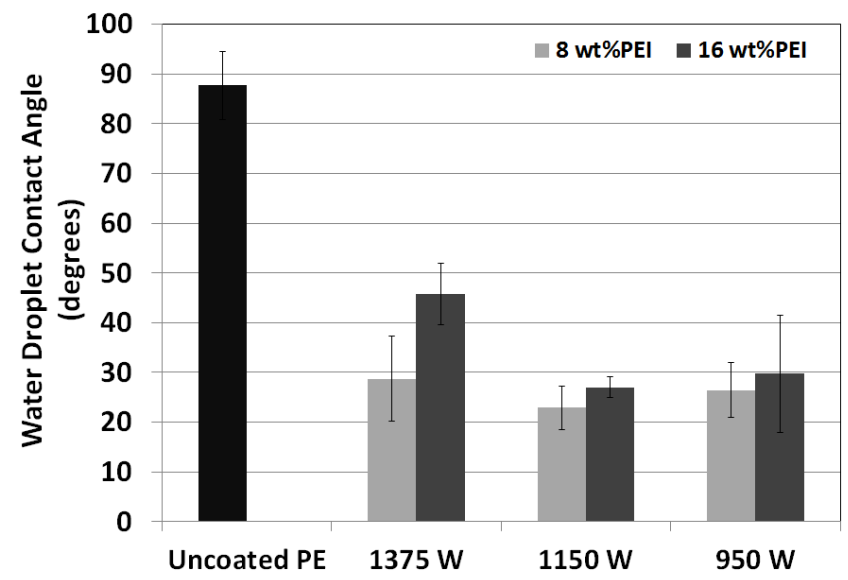

Figure 4: Water droplet contact angle for varying concentration of PEI. Standard deviation measurement is shown with error bars. 


\section{Conclusions and future work}

Bio-compatible coatings were successfully grafted to polyethylene substrates to improve the wettability of the polyethylene. Several characterization methods were utilized to verify the surface chemistry of the substrates was changed. The coatings adhered to the substrate after an ASTM standard tape pull test. The pretreatment allowed for bonding sites on the substrate surface, resulting in stronger coatings. Based on preliminary results, lower input power had a negative effect on the adhesion of the coating to the substrate. Concentration of the polymer precursors did not show an effect based on FTIR and adhesion testing, but investigation of wear properties and plasma parameters would provide a better indication of any trends. Current work is dedicated to establishing relationships between characteristics like thickness, adhesion, and contact angle with the plasma parameters so that design flexibility for the desired application can be achieved.

Further analysis is required to investigate the wear resistance of the atmospheric pressure plasma grafted coatings. Also, instead of using polymers and liquid delivery, the monomer versions of these polymers could be used for coatings that could be more strongly cross-linked. Different delivery methods could be investigated to coat the PE substrates using the monomers; for example, delivering monomers as a vapour through a nozzle at the exit from the plasma jet could improve the coating quality.

\section{References}

[1] Kane, S., Ashby, P.D. \& Pruitt, L. Characterization and tribology of PEGlike coatings on UHMWPE for total hip replacements. Journal of Biomedical Materials Research Part A, 92(4), pp. 1500-1509, 2010.

[2] Hetrick, E. \& Schoenfisch, M., Implant-related infections: active release strategies. Chemical Society Review, 35, pp. 780-789, 2006.

[3] Price, J.S., Tencer, A.F., Arm, D.M. \& Bohach, G.A., Controlled release of antibiotics from coated orthopaedic implants. Journal of Biomedical Materials Research, 30(3), pp. 281-286, 1996.

[4] Sun, L, Berndt, C.C., Gross, K.A. \& Kucuk, A., Material fundamentals and clinical performance of plasma-sprayed hydroxyapatite coatings: a review. Journal of Biomedical Materials Research, 58(5), pp. 570-592, 2001.

[5] Tendero, C., Tixier, C., Tristant, P., Desmaison, J \& Leprince, P., Atmospheric pressure plasmas: A review. Spectrochimica Acta Part B: Atomic Spectroscopy, 61(1), pp. 2-30, 2006. 